\title{
POWer Over Ethernet
}

\section{A SOLUÇÃO DE VANGUARDA NAS COMUNICAÇÕES BASEADAS EM IP}

\section{Introdução}

Mal sabia o Italiano Antonio Santi Giuseppe Meucci na segunda metade do século XIX (1870-1876), que a sua invenção iria alcançar um êxito sem precedente e que mudaria por completo o estilo de vida das gerações vindouras: o "Telettrofono" (telégrafo falante). Porém, e por necessidade, acabou por vender o protótipo e seus respetivos direitos, a Alexander Graham Bell, o qual ficou historicamente conhecido como o inventor do telefone.

Com efeito, o telefone quase que dispensa apresentação. É um dispositivo de telecomunicações que entrou paulatinamente nas vidas das pessoas, seja no lazer, no exercícios das suas profissões, em situações de emergência, em teatro de guerra, num infindável número de situações, e que foi simplesmente planeado para transmitir sons por meio de sinais elétricos em condutores próprios para o efeito (vias telefónicas).

Por definição, é um aparelho eletroacústico que permite a transformação, no ponto emissor, de energia acústica em energia elétrica e, no ponto recetor, a transformação da energia elétrica em acústica, permitindo assim a troca de informações (falada e ouvida) entre dois ou mais assinantes.

Ora, a ideia de Power over Ethernet (PoE) teve, forçosamente, seu marco nos finais do século XIX, quando Alexander Bell ("Bell Telephone Company") teve de refletir/decidir se o telefone a instalar em cada residência, seria alimentado localmente por uma bateria (fonte de energia local), ou se deveria alimentar cada dispositivo telefónico remotamente através de fios condutores. Esta é a noção de Power over Ethernet na sua vanguarda de aplicação no início do século XXI, e objeto de uma breve apresentação, à qual o convido à leitura das restantes secções.

\section{Tecnologia Power Over Ethernet - PoE}

A tecnologia PoE permite que os equipamento e dispositivos que se baseiam em IP ("Internet Protocol"), os quais comunicam na internet, tais como os telemóveis, telefones fixos IP, pontos de acesso à LAN ("Local Area Network") sem fio, câmaras de sistemas de videovigilância, entre outros, recebam simultaneamente a alimentação de energia elétrica e dados, partilhando a mesma infraestrutura de internet existente diminuindo, assim, os custos com a instalação de uma infraestrutura separada para os cabos de energia elétrica, tomadas elétricas, etc.

Apenas no início deste século XXI a tecnologia PoE foi aceite pela indústria (o IEEE), tendo originado a emissão de uma norma internacional (IEEE 802.3af-2003 - POE) intitulada de: Data Terminal Equipment (DTE) Power via Media Dependent Interface (MDI). A referida norma determinou que apenas dois dos quatro pares de cobre entrançados de fios do cabo internet CAT-5 (embora possam operar em cabos par de cobre CAT-3 se a potência requerida for baixa) sejam utilizados para a transmissão da alimentação de energia elétrica, até a um valor de potência de $15,4 \mathrm{~W}$ por porta, aos respetivos equipamentos a serem alimentados.

Porém, os equipamentos e dispositivos mais avançados que se baseiam nesta tecnologia requerem cada vez mais, maior valor de potência que a especificada na norma IEEE 802.3af2003. Para fazer face a esta situação foi desenvolvida uma nova norma com o intuito de melhorar as diretrizes da tecnologia PoE assentes na norma anterior. Assim, recentemente (2009), a publicação da norma IEEE 802.3at2009 (PoE+) promoveu o aumento da capacidade de alimentação. A norma intitulada "Data Terminal Equipment (DTE) Power via Media Dependent Interface (MDI) Enhancements", definiu o valor máximo de $30 \mathrm{~W}$ a ser fornecido por porta. 
Ainda assim existe uma clara tendência e necessidade do mercado em novas tecnologias que permite a transferência de "high-power" (alta potência) simultaneamente com os sinais de dados, utilizando-se os quatro pares do cabo internet a uma distância máxima de 100 metros. Neste contexto pode-se enunciar as seguintes tecnologias (proprietárias) desenvolvidas, as quais são extensões da norma IEEE 802.3at-2009:

- UPoE - Universal Power Over Ethernet: Tecnologia desenvolvida pela Cisco; utilizam-se os quatro pares para transmissão de potência até 60W;

- PoH - Power over HDBaseT: Tecnologia desenvolvida pela HDBaseT Alliance; utilizam-se os quatro pares para transmissão de potência até 100W;

- LTPoE++ - Linear Technology PoE++: Tecnologia desenvolvida pela Linear Technology que fornece quatro diferentes níveis de alimentação (38,7W; 52,7W; 70W e 90W).

Atualmente, e no contexto do panorama nacional de comunicações eletrónicas, vivemos num período de mudança e atualização de especificações técnicas, que vão ao encontro da realidade social e económica do país. Com efeito, a ANACOM (Autoridade Nacional de Comunicações) apresenta no seu site a proposta da 3a Edição do Manual ITED que se encontra em consulta e discussão pública até ao próximo dia 23 de Dezembro do corrente ano de 2013 (http://www.anacom.pt/render.jsp?contentld=1182017,

disponível online em dezembro de 2013). Esta proposta de Manual, embora não seja um Manual de rutura face ao anterior, tem um maior foco sobre a reabilitação e reformulação das frações existentes, propiciando o aparecimento e adaptação das infraestruturas de telecomunicações a novas tecnologias.

Neste contexto, é com naturalidade que na secção 10.9 do documento de proposta da 3áEdição do Manual ITED, seja apresentada a tecnologia PoE como solução a ter em conta em sistemas de comunicações que usem dispositivos baseados na tecnologia IP.

\section{Arquitetura do sistema PoE}

Um sistema Power Over Ethernet (PoE e/ou PoE+) consiste de um único equipamento de fornecimento de energia ("Power Source Equipment" - PSE), um segmento de link (cabo entrançado par de cobre), e um único PD ("Powered Device" ou "Powered End Station", ex: IP camaras de vídeo IP, telefone IP, impressora, computador, etc.) sendo sua arquitetura projetada numa topologia em estrela, conforme os exemplos ilustrados na Figura 1.

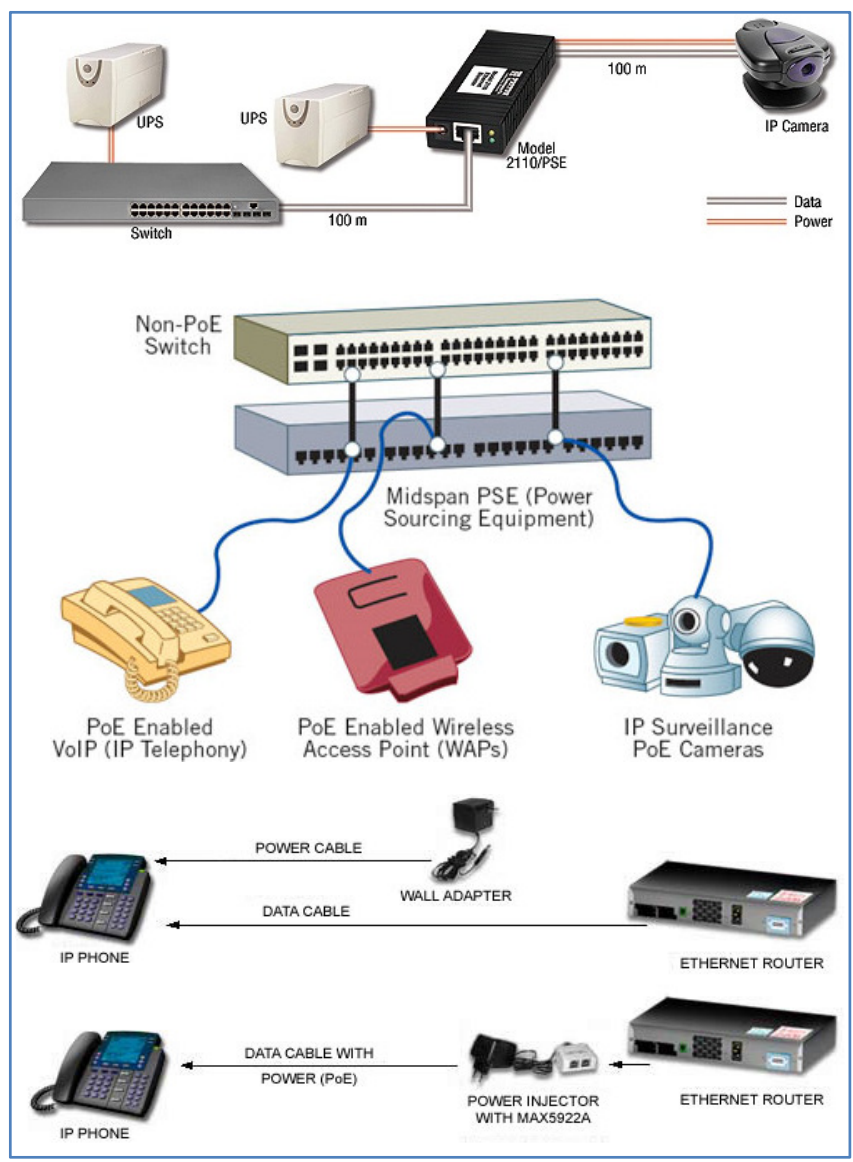

Figura 1 - Exemplos da arquitetura da tecnologia PoE

A especificação IEEE 802.3at-2009 fornece uma rotina denominada de "handshaking" entre o PSE e o PD, antes deste começar a ser alimentado por aquele. Isto assegura que o PSE só aplique a energia DC ("Direct Current") em cada par de cobre quando um dispositivo conectado tenha sido detetado como compatível. 
Basicamente, e de acordo com a especificação IEEE mencionada, a rotina "handshaking" é composta pelas seguintes funções:

- Deteção: Para detetar um equipamento PD, o PSE aplica duas pequenas tensões DC ( $\mathrm{V}^{1}$ e $\mathrm{V}^{2}$ de $2,8 \mathrm{~V}$ até $\left.10 \mathrm{~V}\right)$ no cabo par de cobre extraindo o valor de impedância ("Signature Resistance") a fim de determinar se o equipamento se trata de facto de um PD válido ( valor típico da "signature resistance" entre $19 \mathrm{k} \Omega-26,5 \mathrm{k} \Omega$ );

- Classificação: O PSE tentará classificar o equipamento PD "questionando-o" com uma outra tensão DC no segmento do link. Ao receber esta tensão $\mathrm{DC}$, o equipamento PD envia uma resposta ao PSE com o valor de DC que corresponde à sua classificação. Ao receber a resposta do PD, o PSE identifica e fornece a alimentação requerida.

- Transmissão de alimentação: Dado que alimentação está a ser transmitida, o PSE monitoriza continuamente o consumo DC pelo equipamento PD.

- Desconexão segura: A norma IEEE 802.3at-2009 especifica que quando um PSE parar de receber a "assinatura de alimentação" do PD, o PSE deverá interromper a alimentação, evitando, assim, possíveis danos.

Por vezes os equipamentos terminais PoE (PDs) são instalados em locais de difícil acesso e com distância relativamente elevadas. Respeitando a distância limite imposta por norma ao cabeamento em par de cobre, a instalação entre o PSE e o PD não deverá exceder os 100 metros.

Uma vez que neste tipo de tecnologia se elimina a passagem de cabo de alimentação de energia elétrica aos dispositivos terminais (PD), o grande desafio da aplicação da tecnologia PoE é garantir a alimentação elétrica a PD remotos, nomeadamente em locais fora do limite de distância do cabeamento par de cobre. Mesmo utilizando-se os "Midspans" estes não aumentam a distância da rede de dados. A Figura seguinte reflete um ambiente padrão onde a limitação do cabeamento de cobre é seguido a rigor (exemplo 1 e 2).
Nos casos em que os PDs se situem em distâncias superiores a 100 metros, uma possível solução é a de integrar o cabeamento par de cobre com o cabo de fibra ótica (FO), utilizando-se para tal Conversores de Mídia PoE, conforme representado no exemplo 3 da Figura 2.
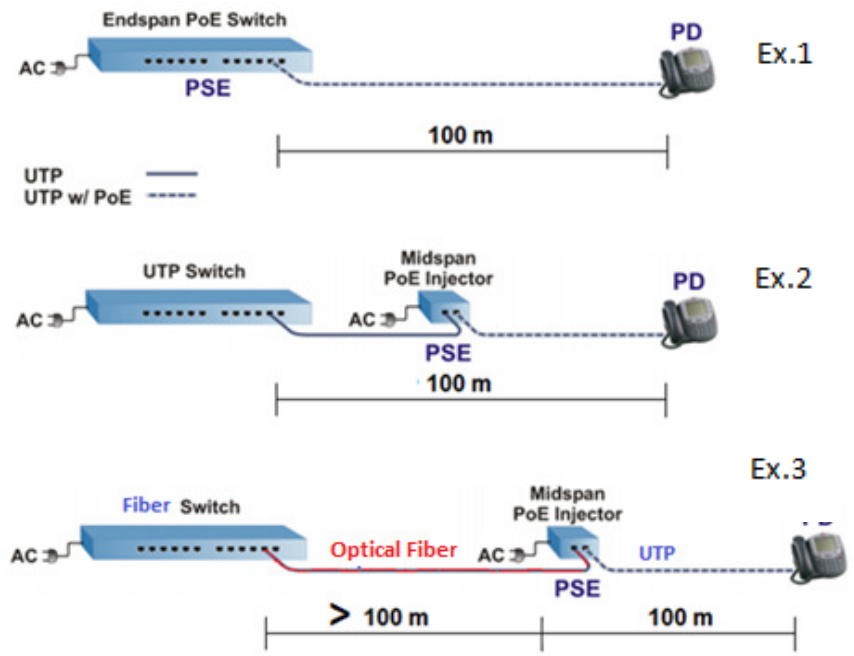

Figura 2 - Representação da distância máxima de alimentação de dispositivos terminais via PoE

Apesar de existirem soluções como Extensores de LAN ("LAN Extenders"), conversores UTP para tecnologia coaxial e tecnologias Wireless, a solução que realmente se apresenta com maior grau de segurança e confiabilidade para assegurar a alimentação aos dispositivos PDs em locais que excedem os 100 metros, é a utilização de Conversores de Mídia PoE, os quais possibilitam a utilização de cabos de fibra ótica.

Conforme representado na Figura 2, os dispositivos PD foram instalados com uma distância onde o limite de 100 metros para o cabeamento UTP foi excedido.

Assim, a gestão dos equipamentos PDs é potenciada com base na implementação de um Conversor de Mídia PoE (PSE) e, consequentemente, o uso de cabo de fibra ótica para conectorizar à LAN.

O cabo de fibra ótica utilizado é estabelecido entre um Switch de FO e termina no Conversor de Mídia PoE, localizado perto de uma fonte de alimentação de energia elétrica, conforme ilustrado na Figura 2, exemplo 3. 
Por sua vez, os dispositivos PD - câmara videovigilância IP, telefone IP, Wireless AP, etc. - são conectados ao Conversor de Mídia PoE, o qual os alimenta em corrente contínua através da utilização de cablagem de par de cobre.

Sucintamente, as principais vantagens da utilização da tecnologia POE são:

- O baixo custo: Com efeito elimina a necessidade de uma infraestrutura de cabeamento para alimentação de cada PD, uma vez que num único cabo é usado para transmissão de energia elétrica e dados;

- Eficiência energética: Devido à facilidade de controlo sobre a alimentação de dispositivos ligados remotamente no edifício ou fração;

- Fiabilidade da instalação: Permite facilmente suportar através dos cabos de comunicação alimentação elétrica socorrida a sistemas críticos, como por exemplo, câmaras de segurança, telefones IP, etc.;

- Flexibilidade: Os equipamentos PDs podem ser localizados independentemente das fontes de energia existentes no edifício ou fração, designadamente em locais de difícil acesso à energia elétrica;
- Gestão: Os equipamentos baseados na tecnologia PoE podem ser facilmente geridos via SNMP (Simple Network Management Protocol).

Conforme referido na secção 10.9.3 da proposta do Manual ITED 3áEdição de dezembro 2013, a utilização de PoE nas Infraestruturas de Telecomunicações em Edifícios (ITED) poderá trazer vantagens acrescidas, nomeadamente quando associado a sistemas complementares de sistemas de segurança (videovigilância) ou na alimentação de pontos de acesso $\mathrm{WiFi}$, conforme representado na Figura seguinte adaptado da proposta de Manual referida.

Como a Figura sugere, o equipamento fonte de energia (PSE) poderá/deverá ficar localizado no ponto de distribuição do fogo (PDF), atualmente e à luz da vigente 2 a Edição do Manual ITED, denominado por ATI. Por motivos extra de segurança, poder-se-á instalar uma fonte socorrida para alimentação (UPS) destes equipamentos. Todos os dispositivos ativos terão de ficar instalados no PDF, sendo a energia injetada na ligação permanente em pares de cobre da rede individual. Com efeito, o RC-PC deverá permitir a passagem de corrente requerida pelos dispositivos PoE, conforme evidencia a Figura 3.

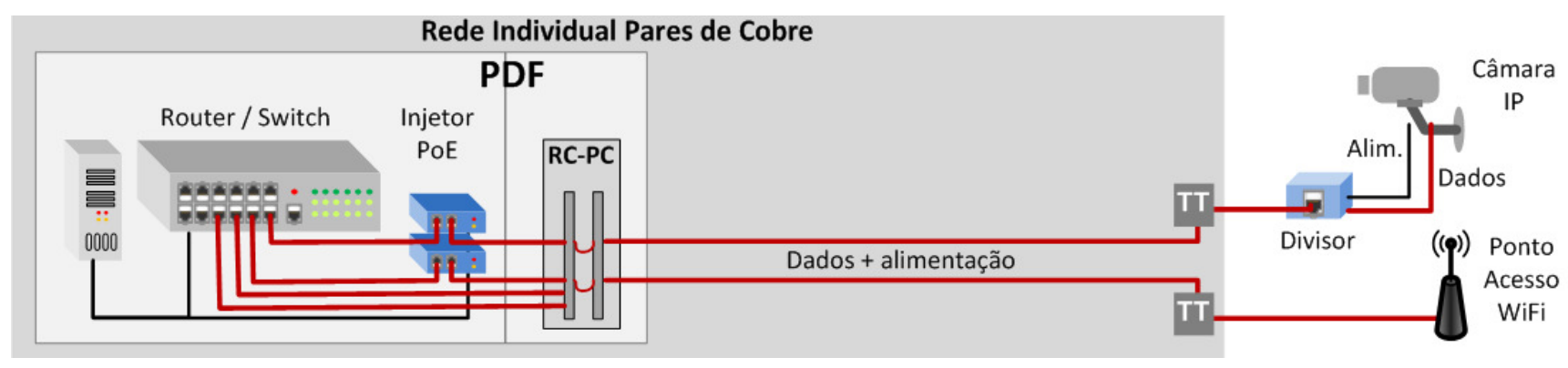

Figura 3 - Aplicação da tecnologia PoE

(Adaptado da proposta de 3a Ed. Do Manual ITED em consulta pública disponível online em dezembro de 2013: http://www.anacom.pt/render.jsp?contentld=1182017) 


\section{Conclusões}

A tecnologia Power over Ethernet - PoE é uma tecnologia emergente, assente em conceitos idealizados e concebidos há quase 150 anos. A relação custo/beneficio é extremamente interessante pelo que se torna cada vez mais numa solução muito atrativa por parte dos fabricantes, aliada à sua extrema comodidade, flexibilidade, recursos avançados de gestão e, não menos importante, segurança.

Esta tecnologia, a par da generalidade das tecnologias de comunicações eletrónicas, está em constante evolução e, consequente, vão aparecendo novas inovações de dispositivos de forma a permitir a transmissão de corrente contínua de maior potência, juntamente com os sinais de dados, sobre a mesma infraestrutura de cablagem.

No entanto, o grande desafio alcançado foi a de permitir alimentar dispositivos PDs através da transmissão de corrente contínua, juntamente com a transmissão de dados, sobre o cabo de par de cobre entrançado, eliminando custos de infraestruturas para o abastecimento de energia elétrica.

De salientar que a tecnologia PoE não tem qualquer influência no desempenho da comunicação de dados existente no mesmo cabo

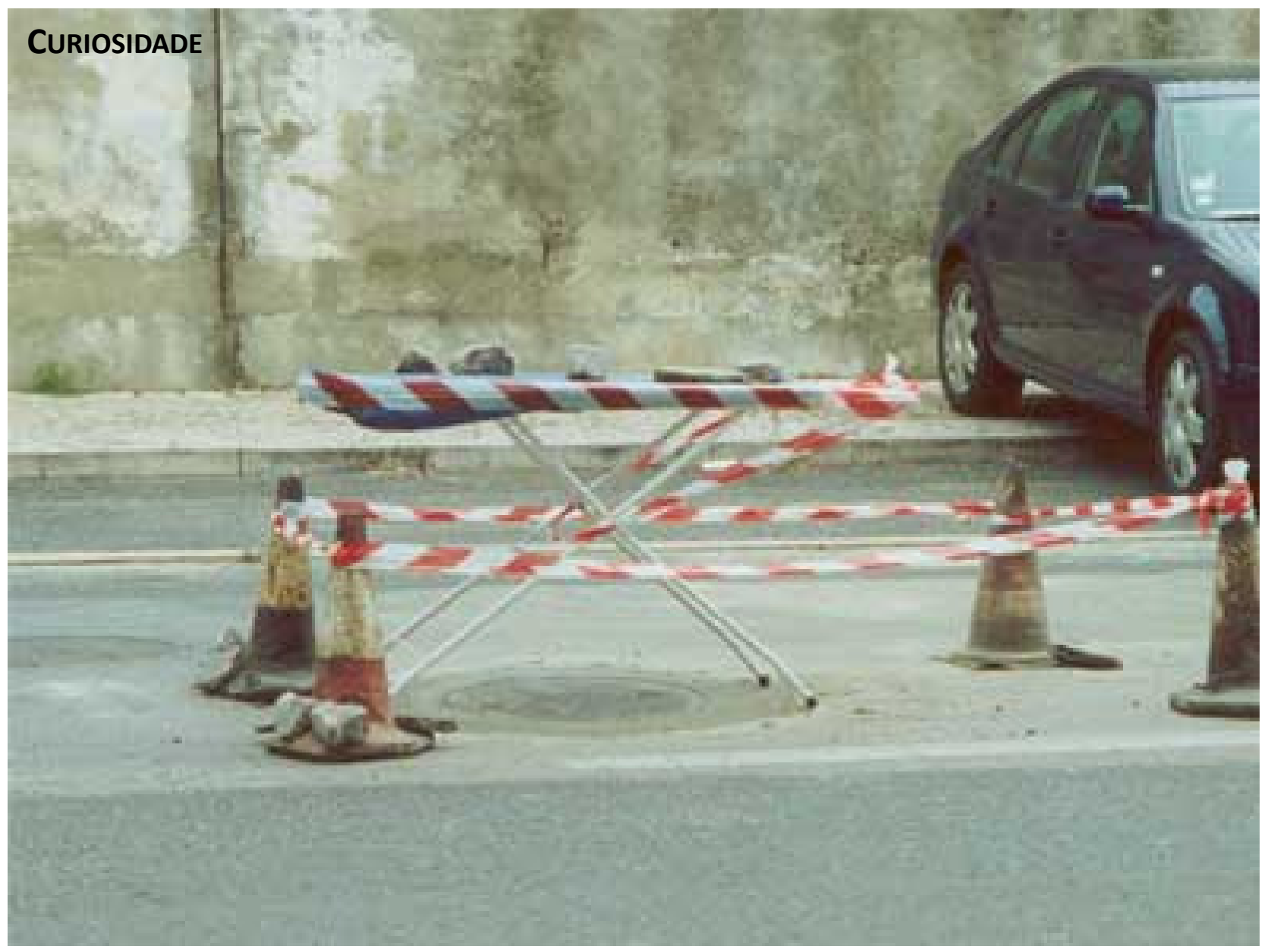

